\title{
Treatment of Burns at the Red Cross War Memorial Childrens Hospital, Cape Town
}

\author{
M. L. BRETT. M.C.S.P., Physiotherapist. Red Cross War Memorial Children's Hospilal
}

The Red Cross Hospital Burns Unit is the centre for the whole of the Western Cape for trealing burns in children up to the age of 13, and over the years, since the hospital opened in 1956, has developed into a very efficicnt and successful unit. The unit is staffed by surgeons, a paediatrician, a plastic surgeon, a sister and Coloured nurses, and during the year 1965. 380 cases were admitted.

\section{ASEPTIC PRECAUTIONS}

Great care is taken to alvoid the introduction of any infection into the unit, or cross infection from one case to another, and all persons cntering the unit itself, not only the cubicles, must wear a surgical mask. The fioors are not swept, in order to avoid circulating dust and germs, but are swabbed twice daily with disinfectant, and the unit is airconditioned. The lemperature of each cubicle is charted daily and can be alicred according to the patient's condition and the weather. The staff are gowned when treating the patients and must wash with germicidal soap bcfore going from one child to the next.

When certain organisms are reported in a burn swab culture (especially betahaemolytic streptococcus or pseudomonas) these particular patients are strictly isolated, and staff entering that cubicle must be gowned and booted as well as masked. In this way infection is kept to a minimum, and one notices the absence of the unpleasant smell usually associated with burn cases. Skin grafting, which is essential in all full thickness burns, will fail if undertaken in the presence of uncontrolled infection, so it is of vital importance that strict aseptic techniques are observed

As the hospital collects patients from such a wide area we tend to have particularly severe burn cases admitted, especially during the winter months when fires are made inside the pondokkics and shacks. These children are often in a poor physical condition prior to being burnt, and though the extent of the burn may seem small they are very liable to develop bronchopneumonia, a common complication in burns.

\section{INITIAL TREATMENT}

On admission, the percentage of the burn is assessed by totalling the area of body surface affected, and the necessary medical treatment is started to combat shock and loss of body fluids. There is no difference in the treatment of a burn caused by fire from one caused by scalding liquids such as water, tea or porridge, except that if in the latter case blisters occur these are left untouched, thereby avoiding the possible introduction of infection through any break in the skin and discouraging further fluid loss. Depending on the location of the burn and the degree of sepsis the patient is nursed either by the "open" or exposed method, which is preferred in most cases, or by the "closed" method, when dressings are applied.

The object of the "open" method is to allow the burns to dry and form their own protective crusts, bacterial colonisation meanwhile being reduced by spraying four-hourly with an anti-bacierial spray such as Polybactin. The "closed" method is used for all burns of the hands and feet, for badly infected cases, and for circumferential burns. Occasionally an old burn case is sent in from the country, having already developed deforming scars and contractures, or webbing of fingers and toes, a result of not bandaging each digit separately. These cases often require years of plastic surgery and much physiotherapy and corrective splinting, to repair an easily preventable deformity.

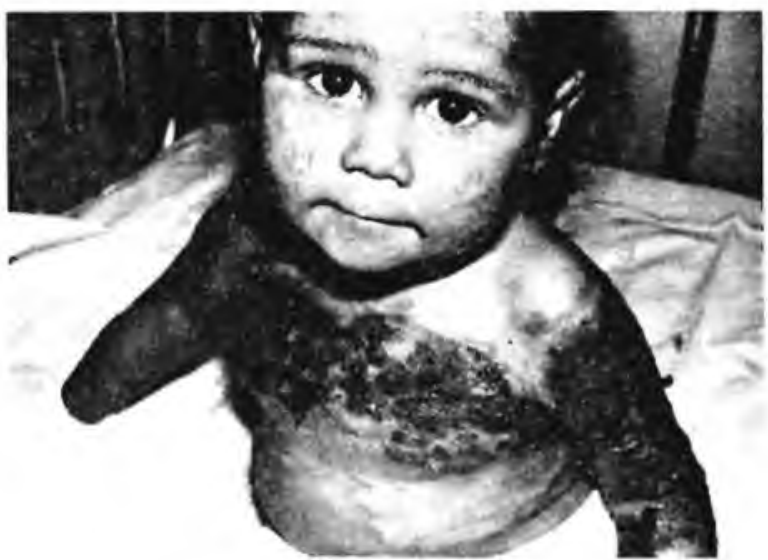

Child wilh a lum over the chest wall, which could cause a chest complication.

\section{CHEST COMPLICATIONS}

In the initial shock stage the first consideration is the preservation of life, which in the field of physiotherapy involves the prevention and treatment of chest complications. Often the burn nay extend over the chest wall, causing the child to breathe shallowly to minimise the movement and consequent pain. This can lead to a collapsed lobe or lung. The skin may be scorched to the consistency of hard leather in a severe burn, with no elasticity at all, and allowing very little expansion of the chest. In these cases the burnt tissue has to be cut into several strips to release the restriction, when the expansion will improve immediately, often by a couple of inches.

Inhalation burns are another great cause of chest complications, and are usually the result of a primus stove exploding in the child's face. Not only will the face be burnt, with the resulting oedema particularly of the lips and nostrils which considerably limits air entry, but the trachea and bronchus may also be aflected due to inhalation of fumes and hot air. This causes inflammation, oedema and sloughing of the muctis membrane so that a chest complication is inevitable, and sometimes a tracheotomy has to be performed.

It is an advantage 10 elevate the foot of the col for a period before treating the child, except in cases of burns of the head and neck, when this position would increase the oedema. It is often necessary to percuss the chest 10 loosen the secretions, and this may have to be done when the patient is nursed by the "open" method, using a couple of sterile dressing towels over the burnt area. The age, condition and intelligence of the patient determines whether the treatment is passive or whether the child will join in, but it is often possible with patience and ingenuity to get a very severely burnt child to co-operate in breathing exercises and blowing games. A suction machine is used during treatment if the chest sounds moist, and always if a tracheotomy has been performed. 
Good positionıng of the patient in the acute stage is also important, to prevent contractures developing, particularly over the flexor aspects of a limb. The shoulder is abducted in a burn of the axillary region-hands are elevated to prevent oedema, and the head of the bed raised and a small pillow placed between the child's scapulae to extend the neck in a burn of the neck and facial areas. A patient with a burn in the groin should spend a good portion of the day in a prone position.

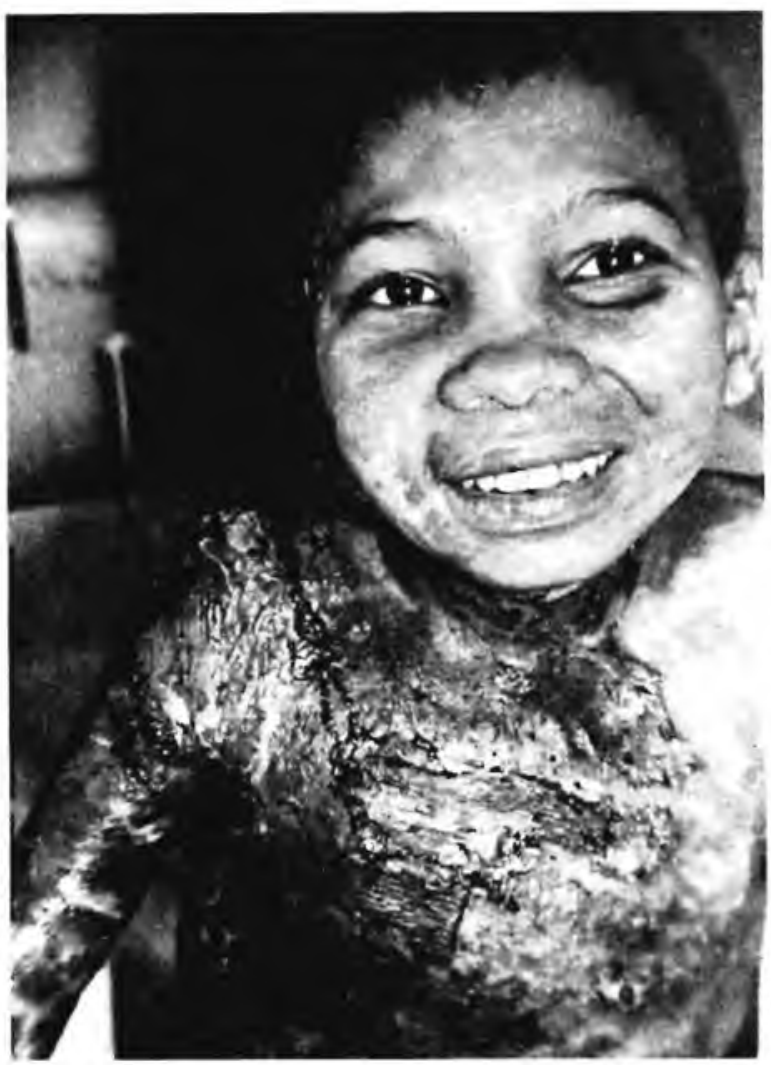

A large area of skill graft showing a good lake.

\section{PRE-OPERATIVE OR INTERMEDIATE STAGE}

After the patient has recovered from the initial shock the physiotherapist is able to concentrate more on the maintenance of mobility and good posture, prevention of contractures and encouragement of healing. Movements are carried out in the fullest possible range within reasonable limits of pain. The burnt child has a long and painful road to recovery, and as most of the patients are very young some of one's previous ideas of treatment have to be modified slightly, and the child's stay in hospital made as happy as possible so that his mind does not bear permanent marks of his ordeal.

Ultra violet light is sometimes requested at this slage, to improve the circulation and speed up the healing process in a slight burn, or perhaps prior to a graft, to help clean and stimulate an infected area. The appropriate erythema dose is given, using a Kromayer lamp which has been found to be far more suitable for use with children than the larger alpine sunlight lamp.

\section{SKIN GRAFTING}

Grafting is usually done within 15-21 days of the burn occurring, depending on how long it takes to get the area cleaned, and the patient's general condition suitable for surgery. The majority of cases require skin grafting, even very small burnt areas. The advantage of this treatment is quicker healing and better skin coverage, eliminating the thin inelastic skin which is produced by spontaneous healing and which tends to ulcerate at a later date. Homografts are often used in an extensive burn when an autograft is difficult to obtain at an early stage due to lack of donor area. This alien skin is rejected by the patient, usually after 10 to 14 days, but it has served the purpose of providing temporary skin coverage, limiting fluid loss, and often stimulates healing in the grafted area.

The grafts are laid on the burnt area in sheet form, leaving no gaps between the strips of skin which would become scarred. Where there is movement such as over the abdomen or chest, or if there is any likelihood of the graft slipping it is stitched into position and left exposed-otherwise it is just bandaged into position with dressings.

The fixation used post-operatively is generally Plaster of Paris as it is difficult to immobilise children sufficiently any other way to prevent them from rubbing off the new graft. The first dressing is left in place for 7 to 8 days. during which time no movements are given which would disturb the skin graft, and the physiotherapist should check whether there is any heavy fixation or tight strapping round the chest area which would necessitate breathing exercises.

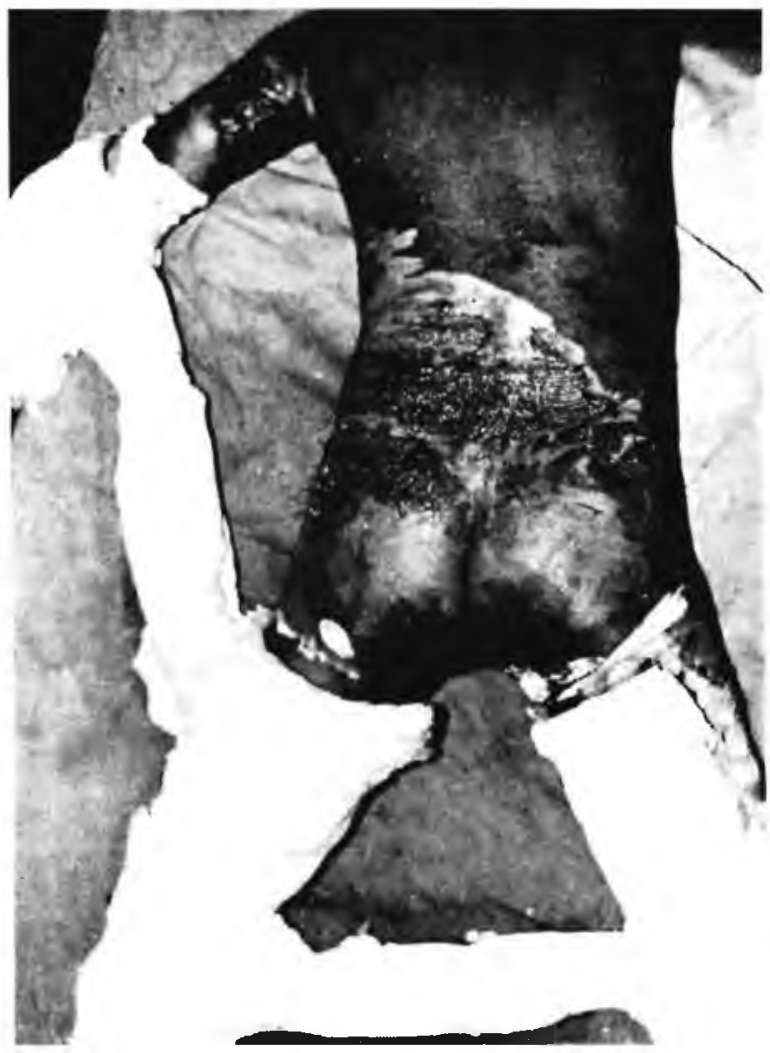

Post operative P.o.P. immobilisation of skin graft on arm and trunk. 


\section{MIRROR SYNKINESES \\ (CONTRALATERAL IMITATIVE ASSOCIATED MOVEMENTS)}

\author{
A. M. K. OBHOLZER, \\ Diskobolos, Kimberley
}

\begin{abstract}
One of the many "nice things" in life is the ability to move the extremities of the left and the right side independently. One can write with the right hand and, at the same time, guide a sandwich to the mouth with the left hand; one can also carry a suitcase with the left hand and beckon a porter with the right one. In this way, one can do many more and some very difficult things like playing the violin. In this case the left hand and the right hand perform completely different difficult movements simultaneously. While doing any of these movements no one does by any means feel like the late and hereto unbeaten king of the jugglers, Rastelli. One takes these abilities for granted, and the reader might wonder why he should be bothered with such an all too natural fact.
\end{abstract}

People who remember their physiology from good old high school or maybe from varsity days will be quick to tell you why one is able to move the limbs independently: "There are two brain halves-hemispheres-a right and a left one. They contain, amongst others, centres for movements. The left hemisphere reigns over the right side of the body and

\section{Burns-Continued from page 4}

All dressings are done in the treatment room, both for aseptic reasons and because this is less traumatic psychologically for the other children in the ward. Large burns are soaked in a mobile bath containing a mixture of water, Cetavlon, and Ringers solution, and a thin perforated pipe circles the base of the bath through which oxygen is bubbled. This helps to loosen the dressings and cleanse the burn. Both bath and treatment room are disinfected between patients.

\section{POST OPERATIVE OR FINAL HEALING STAGE}

After the dressings have been removed and the graft has taken exercises are started again, progressing from gentle to more vigorous movements. Later the children are able to go to the Physiotherapy Department where ball games, pedal toys and other apparatus can be used to gain mobility and confidence.

The grafted area is massaged gently with lanolin, which is mixed with olive oil to a very soft consistency as the skin is both delicate and of a reduced sensitivity at this stage. The grafted skin will tend to contract for anything up to three months after the operation, particularly over the flexor aspects and the palm of the hand, and it requires great supervision on the part of the physiotherapist to prevent deformities developing which may require further surgery. Keloid scarring may occur, particularly in African patients and this has to be excised when it has ceased being active. Night splinting, and "lively" hand splinting for day time use is of great value, and light plaster of Paris splints can be made which may be easily applied by the parents when the child goes home.

Unless the patient lives far away, he attends the hospital as an out-patient, both for physiotherapy treatment and check-up at the Burns Follow-Up Clinic at regular intervals. Many of these children, after sustaining a severe burn, require a series of cosmetic plastic surgery operations which may have to be spread over a period of several years.

In conclusion, it can be seen that the physiotherapist has a most important part to play in the general team work of rehabilitating the burnt child, and her work is complementary to that of the surgeon in restoring the patient to a normal life. the right one over the left side. This is due to the fact that the Pyramidal Tracts, which transfer the innervating impulses from the motor centres of the brain to the muscles of the body, cross sides in the Decussation. The two hemispheres and their Pyramidal Tracts work independently. So, why should the right hand/arm not be able to carry out a movement different from that of the left-even if these movements take place simultaneously?"'

Yes, indeed, why not-if the premise to the conclusion is correct, i.e. if the right hemisphere is really only responsible for the movements of the left side of the body and vice versa.

There is, however, some reason for doubt concerning the correctness of this stated premise because:

\section{Mirror Synkineses Associated with Pathological Conditions}

There are people who are in fact unable to move the upper extremities independently from each other. This phenomenon we find not so rarely amongst spastic hemiplegias. In these cases a palsied hand/arm which is otherwise unable to move at all, may do so if the healthy hand/arm does a movement against strong resistance (Obholzer) ${ }^{\mathbf{1}}$. These "associated movements" might be so slight that they are not really a movement yet but only an increase in muscle tone. But even then we can regard this as an abortive copy of the movement of the contralateral side.

If the copied movement is well executed then we speak of a Mirror Synkinesis (M.S.).

There is also a special condition called Klippel-Feil syndrome which shows Mirror Synkineses (M.S.). Here, mainly the cervical vertebrae are misformed, causing neck movements to be restricted. Bauman ${ }^{2}$ studied six such cases, and found M.S. in four out of the six cases. Before Bauman, papers on about 30 cases of Klippel-Feil syndrome had been published, but nothing was mentioned re: M.S. It is believed that M.S. were not noticed in these cases only because no one was on the lookout for them, the connection between Klippel-Feil syndrome and M.S. not being known at that time. M.S. can often easily be disguised and thus overlooked.

There is also a familial trend which is noticeable in M.S. An example: $\mathrm{Smith}^{3}$ reporting of a 23-year old father and his one-year old son, both showing M.S.

A case where M.S. were linked with metabolic disturbances (phenylpyruvicaciduria) has been reported by Friedman and Levinsohn ${ }^{4}$.

M.S. usually exists from birth, and sufferers "learn" to suppress them in later life more and more. The innate condition, however persists throughout life.

\section{M.S. in Neurologically Normal Cases}

There are also neurologically normal persons who are unable to move the upper extremities independently. There is the case of a right-handed person who when he wrote with the right hand, copied the movement with the left hand. When he tried to stop the movement of the left hand by sitting on it, he was unable to continue writing with the right hand. If, however, he took a pen in the left hand as well and started writing with the right hand, the left hand copied what the right hand wrote ... but mirrorwise, i.e. from right to left and with inverted letters. As one can read what the left hand had written only with the help of a mirror, we call this type of writing "Mirror Writing" (Critchley", This man, I can assure you was not too happy about his "gift". When he carried a bucket of water in his left hand and wanted to open a door with the right hand, he had to put the bucket down first, otherwise, he would have dropped the bucket when turning the handle.

We also know of the case of a young man with this type of M.S., reported by Burr and Crow ${ }^{6}$. He entered the navy but proved to be unable to climb a ladder, as every time he released a bar with one hand in order to grab the next higher one, the other hand also opened ... and he fell down. 